\title{
DETECTION OF SPECTRAL EVOLUTION IN THE BURSTS EMITTED DURING THE 2008-2009 ACTIVE EPISODE OF SGR J1550-5418
}

\author{
Andreas von Kienlin ${ }^{1}$, David Gruber ${ }^{1}$, Chryssa Kouveliotou ${ }^{2}$, Jonathan Granot ${ }^{3}$, Matthew G. Baring ${ }^{4}$, \\ Ersin GöĞÜş ${ }^{5}$, Daniela Huppenkothen ${ }^{6}$, Yuki KaneKo ${ }^{5}$, Lin Lin ${ }^{5}$, Anna L. Watts ${ }^{6}$, Narayana P. Bhat ${ }^{7}$, \\ Sylvain Guiriec ${ }^{7,8}$, Alexander J. van Der Horst ${ }^{6,9}$, Elisabetta Bissaldi ${ }^{1,10}$, Jochen Greiner $^{1}$, Charles A. Meegan ${ }^{9}$, \\ William S. Paciesas ${ }^{7}$, Robert D. Preece ${ }^{7}$, And Arne RaU ${ }^{1}$ \\ ${ }^{1}$ Max-Planck-Institut für extraterrestrische Physik, Giessenbachstraße, D-85748 Garching, Germany; azk@mpe.mpg.de \\ ${ }^{2}$ Space Science Office, VP62, NASA/Marshall Space Flight Center, Huntsville, AL 35812, USA \\ ${ }^{3}$ The Open University of Israel, 1 University Road, POB 808, Ra' anana 43537, Israel \\ ${ }^{4}$ Department of Physics and Astronomy, Rice University, MS-108, P.O. Box 1892, Houston, TX 77251, USA \\ ${ }^{5}$ Faculty of Engineering and Natural Sciences, Sabanc1 University, Orhanl1-Tuzla, İstanbul 34956, Turkey \\ ${ }^{6}$ Astronomical Institute "Anton Pannekoek," University of Amsterdam, Postbus 94249, NL-1090 GE Amsterdam, The Netherlands \\ ${ }^{7}$ Center for Space Plasma and Aeronomic Research, University of Alabama in Huntsville, 320 Sparkman Drive, Huntsville, AL 35805, USA \\ 8 NASA Goddard Space Flight Center, Greenbelt, MD 20771, USA \\ ${ }^{9}$ Universities Space Research Association, NSSTC, 320 Sparkman Drive, Huntsville, AL 35805, USA \\ ${ }^{10}$ Institute of Astro and Particle Physics, University of Innsbruck, Technikerstrasse 25, A-6176 Innsbruck, Austria \\ Received 2012 March 27; accepted 2012 June 20; published 2012 August 6
}

\begin{abstract}
In early 2008 October, the soft gamma repeater SGR J1550-5418 (1E 1547.0-5408, AX J155052-5418, PSR J1550-5418) became active, emitting a series of bursts which triggered the Fermi Gamma-ray Burst Monitor (GBM) after which a second especially intense activity period commenced in 2009 January and a third, less active period was detected in 2009 March-April. Here, we analyze the GBM data for all the bursts from the first and last active episodes. We performed temporal and spectral analysis for all events and found that their temporal characteristics are very similar to the ones of other SGR bursts, as well the ones reported for the bursts of the main episode (average burst durations $\sim 170 \mathrm{~ms}$ ). In addition, we used our sample of bursts to quantify the systematic uncertainties of the GBM location algorithm for soft gamma-ray transients to $\lesssim 8^{\circ}$. Our spectral analysis indicates significant spectral evolution between the first and last set of events. Although the 2008 October events are best fitted with a single blackbody function, for the 2009 bursts an optically thin thermal bremsstrahlung is clearly preferred. We attribute this evolution to changes in the magnetic field topology of the source, possibly due to effects following the very energetic main bursting episode.
\end{abstract}

Key words: pulsars: individual (SGR J1550-5418, 1E 1547.0-5408, PSR J1550-5418) - stars: neutron X-rays: bursts

Online-only material: color figures

\section{INTRODUCTION}

Soft gamma repeaters (SGRs) together with anomalous X-ray pulsars (AXPs) comprise a small group of X-ray pulsars with many observational similarities. They have slow spin periods clustered in a narrow range $(P \sim 2-12 \mathrm{~s})$ and relatively large period derivatives $\left(\dot{P} \sim 10^{-13}-10^{-10} \mathrm{~s} \mathrm{~s}^{-1}\right)$. Their inferred surface dipole magnetic fields of $10^{14}-10^{15} \mathrm{G}$ (Kouveliotou et al. 1998, 1999) place these sources at the extreme end of the distribution of magnetic fields in astrophysical objects. Such objects were predicted theoretically by Duncan \& Thompson (1992), Paczynski (1992), and by Usov (1992); the former team named such high $B$-field sources "magnetars." Phenomenologically, a distinct difference between AXPs and SGRs is manifested by their bursting activity behavior. SGRs have been observed to undergo active episodes with a multitude of short, soft bursts with energies upward of $10^{36} \mathrm{erg}$, reaching over $10^{45} \mathrm{erg}$ in the case of the very rare giant flares. AXPs, on the other hand, are not such prolific and energetic bursters. As a result, SGRs were historically discovered from their burst activity, while AXPs were set aside from rotation-powered X-ray pulsars by their timing properties with no bursts. This changed in 2002, when Gavriil et al. (2002) discovered bursts from the AXP 1E1048.1-5937; today almost all AXPs have also been shown to emit
SGR-like bursts, albeit fainter. As a group, magnetars are persistent $\mathrm{X}$-ray emitters with X-ray luminosities ranging between $10^{32}$ and $10^{36} \mathrm{erg} \mathrm{s}^{-1}$, larger than those obtained by rotational energy losses, supporting the hypothesis that magnetic field dissipation powers their X-ray emission. Alternative models, such as, e.g., accretion from a fossil supernova fallback disk, have also been invoked to explain the magnetar emission; for comprehensive reviews, see Woods \& Thompson (2006) and Mereghetti (2008), and references therein.

SGR J1550-5418 is a source that has undergone multiple identity changes. The source was discovered in 1980 with the Einstein observatory (Einstein source: 1E 1547.0-5408) during a search for X-ray counterparts of $C O S-B$ unidentified $\gamma$-ray sources (Lamb \& Markert 1981). The Advanced Satellite for Cosmology and Astrophysics (ASCA) confirmed the detection during a Galactic Plane survey in 1998 (ASCA source: AX J155052-5418; Sugizaki et al. 2001). Gelfand \& Gaensler (2007) proposed, on the basis of XMM-Newton and Chandra X-Ray Observatory (Chandra) observations in 2004 and 2006, a potential magnetar/supernova remnant (SNR) association between this X-ray source and the Galactic radio shell G327.24-0.13. The location in the center of the SNR candidate, the relatively soft X-ray spectrum, and the X-ray variability favored a magnetar model explanation; 
however the $X M M$ observation only set an upper limit for the peak-to-peak pulsed fraction. The crucial piece of evidence was finally provided by radio observations (PSR J1550-5418; Camilo et al. 2007). Their measurement of the spin period of $2.07 \mathrm{~s}$ and period derivative of $2.3 \times 10^{-11} \mathrm{~s} \mathrm{~s}^{-1}$ led to an estimate for the surface magnetic dipole field of $B \sim$ $2.2 \times 10^{14} \mathrm{G}$, which confirmed the magnetar nature of the source.

Due to the lack of bursting activity, the source was initially characterized as an AXP (Camilo et al. 2007). However, in $2008 / 2009$ the source entered an extremely active period emitting a plethora of SGR-like bursts in three active episodes, of which the first and the last were the least prolific. Based on the main episode behavior, during which several hundreds of bursts were emitted during a $24 \mathrm{hr}$ period, similar to the burst "storms" of SGRs 1627-41 (Esposito et al. 2008), 1900+14 (Göğüş et al. 1999; Israel et al. 2008), and 1806-20 (Göğüş et al. 2000), the source was renamed as SGR J1550-5418 (Palmer 2009; Kouveliotou et al. 2009). A detailed study of the 2008 October Swift X-ray bursts from SGR J1550-5418 is presented in Israel et al. (2010). A detailed analysis of $\sim 200$ bursts detected within a few hours on January 22 with the International GammaRay Astrophysics Laboratory (INTEGRAL) is presented in Mereghetti et al. (2009) and Savchenko et al. (2010). Finally, Terada et al. (2009) present the analysis of over 250 bursts (50 keV to $5 \mathrm{MeV}$ ) detected with the Suzaku/Wide-band Allsky Monitor (WAM), while the observations of the Swift-BAT, Konus-Wind, and RHESSI are published by Gronwall et al. (2009), Golenetskii et al. (2009), and Bellm et al. (2009), respectively.

Chandra and RXTE observations, performed after the 2008 and 2009 outbursts, provided evidence of a decoupling between magnetar spin and radiative properties ( $\mathrm{Ng}$ et al. 2011). The pulsar spin-down was observed to increase by a factor of 2.2 during the first 2008 event, in the absence of a corresponding spectral change. During the more energetic 2009 events, no such variation was found. A comprehensive analysis of multiple instrument X-ray data by Bernardini et al. (2011) recorded since the 1980 discovery of the source showed that the X-ray flux history can be grouped into three levels: low, intermediate, and high. The observed persistent spectra harden when transitioning from the low to the high state, with the power-law (PL) component becoming flatter and the temperature of the blackbody (BB) increasing. During the high flux state, the pulsed fraction decreases with energy and shows an anti-correlation with the $\mathrm{X}$-ray flux.

The Gamma-ray Burst Monitor (GBM) onboard Fermi detected all three active episodes of the source. A detailed temporal and (integrated) spectral study of the main (second) burst episode (2009 January 22-29) comprising 286 bursts was published by van der Horst et al. (2012). A search in the Fermi-LAT data in the $0.1-10 \mathrm{GeV}$ energy range did not reveal significant gamma-ray emission (Abdo et al. 2010). During this episode, the first GBM trigger on 2009 January 22 showed a $\sim 150$ s long persistent emission with intriguing timing and spectral properties. Kaneko et al. (2010) identified coherent pulsations up to $\sim 110 \mathrm{keV}$ at the spin period of the neutron star and an additional (to a PL) BB component required to model the enhanced emission spectra. The favored emission scenario is a surface hot spot with the dimensions of the magnetically confined plasma near the neutron star surface (roughly a few $\times 10^{-5}$ of the neutron star area). Tiengo et al. (2010) claimed two best-fit source distances of $3.9 \mathrm{kpc}$ and $\sim 5 \mathrm{kpc}$, using the X-ray rings around
SGR J1550-5418 produced by dust scattering. Here, we adopt a distance of $5 \mathrm{kpc}$.

Such an extensive burst activity with hundreds of bursts within a few days has only been observed from three other sources in the past: SGRs 1806-20, 1900+14, and 1627-41. However, lower level burst activity prior to the main bursting episode was only seen from SGRs 1900+14 and 1806-20. Detailed spectral analyses of the bursts from these sources showed that the spectral properties of the earlier bursts were not distinct from those occurring during their peak activity episodes (Göğüş et al. 1999, 2000). In contrast, in SGR J1550-5418 the "before" and "after" burst episodes (2008 October and 2009 March-April) were significantly different from the main "storm" of activity: the burst rates and event intensities were much lower. We report here our spectral analysis results of the first and last bursting episode from SGR J1550-5418, where we see for the first time a clear distinction between the spectral shapes of events detected before and after the main active episode of the source. Therefore, we find here an intriguing new type of behavior that was not observed before in other SGRs. In Section 2, we present an overview of the source burst activity as seen with GBM, while Section 3 presents detailed temporal and spectral analysis of the bursts. Since this is the first soft transient source detected with GBM, we discuss here also the GBM location accuracy for soft sources. We discuss our results, and in particular the implications of the spectral differences between the two episodes, in Section 4.

\section{OVERVIEW OF THE GBM OBSERVATIONS OF SGR J1550-5418 BURSTS}

On 2008 October 3, GBM triggered four times on soft bursts consistent with the same Galactic location (von Kienlin $\&$ Briggs 2008). The source triggered also the Swift satellite (Krimm et al. 2008a, 2008b) approximately 25 minutes after the first GBM trigger, enabling an accurate location and the subsequent identification of the source with the magnetar candidate 1E 1547.0-5408 (Rea et al. 2008). The GBM locations were all consistent with the 1E 1547.0-5408 position (see Section 3.2). None of the GBM bursts on 2008 October was detected with Swift, as the two spacecrafts were on opposite sides of the Earth; an untriggered burst search did not reveal any additional bursts. The last GBM burst activity was recorded on 2008 October 10, 12:53:38 UT (van der Horst \& Briggs 2008).

The source became active again on 2009 January 22, in a second period of extremely high bursting activity (Connaughton \& Briggs 2009; von Kienlin \& Connaughton 2009; Kouveliotou et al. 2009; Mereghetti et al. 2009), with several short and very bright bursts occurring at intervals of a few seconds to about $150 \mathrm{~s}$ between average bursts. In the next $\sim 30 \mathrm{~d}$, the instrument triggered on 117 discrete bursts through February 24, of which 15 were extremely intense, saturating the data rate. During the first $24 \mathrm{hr}$ (January 22) only 41 triggers were recorded as GBM flight software requires a minimum of $596 \mathrm{~s}$ between triggers. A search for untriggered events revealed a total of $\sim 450$ bursts during this $24 \mathrm{hr}$ period (Kaneko et al. 2010; van der Horst et al. 2012). The source remained active until 2009 February 24 , albeit with a decreased rate of roughly two triggers per day, with exceptionally intense bursts occurring about once per week.

After a month of quiescence, the source entered a third period of medium activity, starting on 2009 March 22, 18:56:23.75 UT and lasting until 2009 April 17. During this period, the GBM triggered 14 times with $\sim 4 \mathrm{hr}$ to $\sim 6 \mathrm{~d}$ between individual triggers. The search for untriggered outbursts did not reveal 


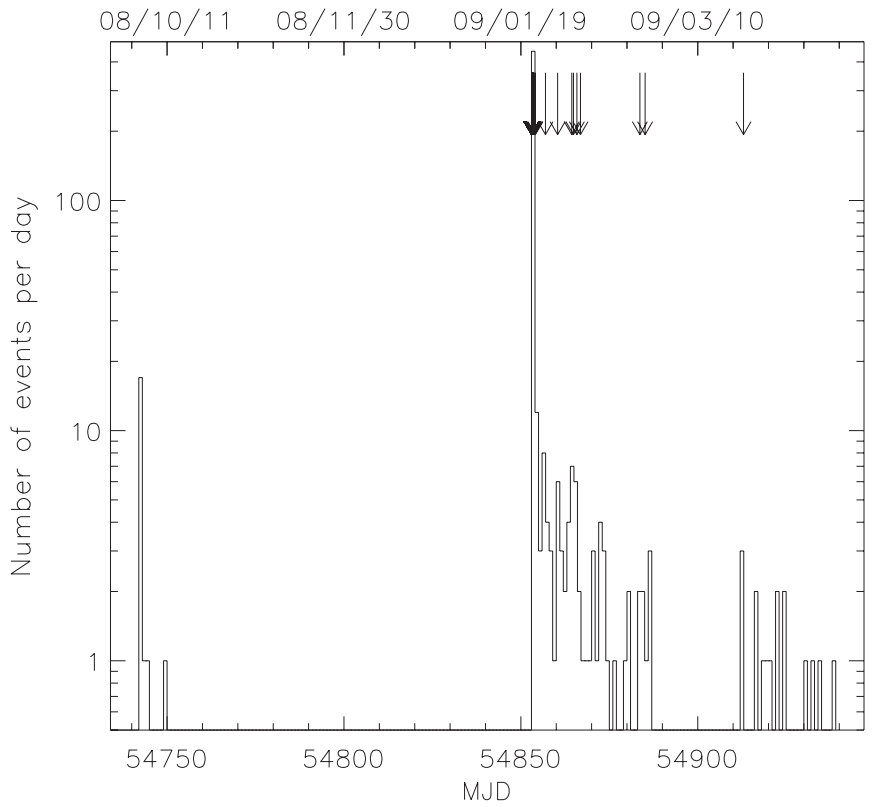

Figure 1. Number of SGR J1550-5418 bursts per day over $\sim 210 \mathrm{~d}$. Three active periods are clearly visible, with an activity peak at the beginning of the second period (MJD 54850). Arrows indicate times of 61 exceptionally bright events (note that there are significant arrow overlaps in the figure).

any additional events. Only one bright burst caused a saturation of the onboard science data bus. During this last period, the mean burst fluence and peak flux were brighter compared to the 2008 October activity, but fainter when compared to the 2009 January-February period. The three periods are shown in Figure 1 as the number of events per day, exhibiting a distinct peak at the beginning of the second activity period (MJD 54850).

Table 1 shows the list of GBM SGR J1550-5418 triggers and bursts during the 2008 October and 2009 March-April activity periods. The first trigger on 2008 October 3 includes 15 individual bursts. None of these bursts showed emission in the two GBM bismuth germanate (BGO) detectors (0.2-40 MeV), so the data from these detectors were excluded from our spectral analysis.

The search for untriggered events was performed from 2008 September 22 to October 13 and from 2009 January 15 to April 22 (2008 October 6 and 2009 March 12 and 13 were left out due to data problems), thus covering part of the first gap and the full second gap of bursting activity visible in Figure 1. For the 93 d, from 2008 October 14 until 2009 January 14, left out by the untriggered burst search, we can only exclude the occurrence of additional events by the fact that GBM and any other instrument did not trigger on bursts from this source. This is corroborated by the finding that all bursts detected by the untriggered bursts search during the activity periods presented here did trigger GBM or occurred during the $\sim 600 \mathrm{~s}$ time period where GBM switched into trigger mode.

\section{DATA ANALYSIS}

The Fermi-GBM instrument comprises two different types of scintillation detectors. Burst locations and the low-energy part of spectra ( $8 \mathrm{keV}$ to $1 \mathrm{MeV}$ ) are provided by an array of 12 thalliumactivated sodium iodide (NaI) scintillator detectors (numbered from $\mathrm{NaI} 0$ to $\mathrm{NaI} 11$ ). The two $\mathrm{BGO}$ detectors extend the spectral coverage to $\sim 40 \mathrm{MeV}$ (for a detailed overview of the instrument and its capabilities, see Meegan et al. 2009). Here, we use only the spectral data below $200 \mathrm{keV}$, since none of the bursts examined showed emission at higher energies.

GBM provides several trigger data types (Meegan et al. 2009): the continuous high spectral resolution data CSPEC, with 128 energy channels, logarithmically spaced over the energy range of the $\mathrm{NaI}$ detectors and $1.024 \mathrm{~s}$ time resolution; the continuous high time resolution CTIME data, binned at $64 \mathrm{~ms}$, with only eight channel spectral resolution; and the time-tagged event (TTE) data, which we used for the analysis of the relatively short SGR bursts. The TTE data consist of a time-tagged photon event list with a temporal resolution as low as $2 \mu$ s and the same spectral resolution as the CPSEC data. The TRIGDAT data, the first burst information, quickly downlinked after a burst trigger, comprise information on background rates, the burst intensity, hardness ratio, localization, and classification, which is needed for the on-ground localization.

\subsection{Data Selection Criteria}

Fermi is operated in sky-scanning survey mode, with the viewing direction rocking to north and south by $35^{\circ}$ from the zenith on alternate orbits, at a slew rate up to several degrees per minute. This operation mode causes a continuous change of the angle between the source and the detector normal. We have analyzed here bursts viewed by detectors with source aspect angles less than $40^{\circ}$. This selection is justified by the softer SGR spectra and the detector effective area, which decreases more rapidly with source angle for softer photons (Bissaldi et al. 2009). None of the selected detectors was subject to blockage from other parts of the Fermi spacecraft.

Figure 2 shows a $200 \mathrm{~s}$ section of the TTE data light curve during the first trigger bn081003.377 in $64 \mathrm{~ms}$ bins. As indicated on the figure, we find eight untriggered bursts during this period, in addition to the one that triggered the instrument at $\sim 0 \mathrm{~s}$. Table 1 lists the start times of all individual bursts related to the trigger time, together with the detector numbers and data types used in our analysis. In this first trigger, detectors $\mathrm{NaI} 1$ and 2 had the smallest source aspect angle during the $\sim 330$ s TTE data period. At times starting after the TTE data type ends until the end of the trigger mode at $\sim 600 \mathrm{~s}$, only continuous CSPEC and CTIME data are recorded, and detector NaI 5 also fulfills the $<40^{\circ}$ selection criterion.

Figure 3 exhibits individual bursts from the two active periods. The first panel of the top row shows trigger bn081003.377, which triggered GBM at $T_{0}=$ 9:03:06 UTC on 2008 October 3 (burst 2 of Table 1; see also Figure 2). The next two panels show untriggered bursts: the first event at $T_{0} \sim-19.9 \mathrm{~s}$, which was too weak to trigger GBM, and bursts 7 and 8, observed at $T_{0}+117.5 \mathrm{~s}$. The light curve of the brightest event during this period, burst 20, which triggered GBM one day later, is shown in the fourth panel. The shape, intensity, and duration of all these events are typical for all other bursts observed during the 2008 October activity period.

During the 2009 March-April period, only 15 bursts (also listed in Table 1) were observed, including two very bright bursts with single detector peak count rates $>1 \times 10^{5}$ count s $^{-1}$. At these high count rates, the performance of GBM is affected by dead time and pulse pile-up as described in detail in Meegan et al. (2009). The light curve of trigger bn090322.944 (burst 24 of Table 1, shown in the leftmost panel of the bottom row in Figure 3), saturated GBM for $\sim 100 \mathrm{~ms}$. The flat plateau from $T_{0}+\sim 80 \mathrm{~ms}$ to $T_{0}+\sim 185 \mathrm{~ms}$ is caused by the clipping of TTE events at the maximum rate of the High Speed Science Data Bus (HSSDB). Burst 29 (trigger bn090330.237), shown 
Table 1

SGR J1550-5418 Trigger Times and Durations

\begin{tabular}{|c|c|c|c|c|c|c|c|c|}
\hline \multirow{2}{*}{$\begin{array}{l}\text { Burst } \\
\text { No. }\end{array}$} & \multirow{2}{*}{$\begin{array}{c}\text { Trigger }^{\mathrm{a}} \\
\text { No. }\end{array}$} & \multirow{2}{*}{$\begin{array}{c}\text { Trigger Time } \\
\text { (UTC) }\end{array}$} & \multirow{2}{*}{$\begin{array}{l}\text { Start }^{\mathrm{b}} \\
(\mathrm{s})\end{array}$} & \multirow{2}{*}{$\begin{array}{l}\text { Stop }^{b} \\
\text { (s) }\end{array}$} & \multicolumn{2}{|c|}{ Duration } & \multirow{2}{*}{$\begin{array}{c}\text { Detector } \\
\text { Nos. }\end{array}$} & \multirow{2}{*}{$\begin{array}{l}\text { Data } \\
\text { Type }\end{array}$} \\
\hline & & & & & $\begin{array}{c}T_{90} \\
(\mathrm{~ms})\end{array}$ & $\begin{array}{c}T_{50} \\
(\mathrm{~ms})\end{array}$ & & \\
\hline 1 & bn081003377U & 9:03:06 & -19.888 & -19.840 & $160 \pm 29$ & $72 \pm 11$ & 1,2 & TTE/CTTE \\
\hline 2 & bn081003377 & 9:03:06 & -0.048 & 0.128 & $276 \pm 53$ & $120 \pm 25$ & 1,2 & TTE/CTTE \\
\hline 3 & bn081003377U & 9:03:06 & 10.112 & 10.496 & $672 \pm 72$ & $320 \pm 72$ & 1,2 & TTE/CTTE \\
\hline 4 & bn081003377U & 9:03:06 & 27.168 & 27.264 & $68 \pm 25$ & $28 \pm 11$ & 1,2 & TTE/CTTE \\
\hline 5 & bn081003377U & 9:03:06 & 89.568 & 89.664 & $128 \pm 23$ & $56 \pm 23$ & 1,2 & TTE/CTTE \\
\hline 6 & bn081003377U & 9:03:06 & 109.504 & 109.728 & $424 \pm 72$ & $120 \pm 18$ & 1,2 & TTE/CTTE \\
\hline 7 & bn081003377U & 9:03:06 & 117.488 & 117.568 & $64 \pm 23$ & $24 \pm 18$ & 1,2 & TTE/CTTE \\
\hline 8 & bn081003377U & 9:03:06 & 117.904 & 118.048 & $104 \pm 34$ & $56 \pm 11$ & 1,2 & TTE/CTTE \\
\hline 9 & bn081003377U & 9:03:06 & 149.824 & 149.952 & $136 \pm 18$ & $64 \pm 11$ & 1,2 & TTE/CTTE \\
\hline 10 & bn081003377U & 9:03:06 & 240.736 & 240.768 & $\ldots{ }^{c}$ & $\ldots{ }^{c}$ & 1,2 & TTE/CTTE \\
\hline 11 & bn081003377U & 9:03:06 & 306.760 & $\ldots$ & $\ldots$ & $\ldots$ & $1,2,5$ & CTIME \\
\hline 12 & bn081003377U & 9:03:06 & 321.090 & $\ldots$ & $\ldots$ & $\ldots$ & $1,2,5$ & CTIME \\
\hline 13 & bn081003377U & 9:03:06 & 411.780 & $\ldots$ & $\ldots$ & $\ldots$ & $1,2,5$ & CTIME \\
\hline 14 & bn081003377U & 9:03:06 & 468.440 & $\ldots$ & $\ldots$ & $\ldots$ & $1,2,5$ & CTIME \\
\hline 15 & bn081003377U & 9:03:06 & 584.137 & 585.161 & $\ldots$ & $\ldots$ & $1,2,5$ & CSPEC \\
\hline 16 & bn081003385 & $9: 14: 00$ & -0.168 & 0.008 & $108 \pm 18$ & $56 \pm 34$ & $1,2,5$ & TTE/CTTE \\
\hline 17 & bn081003385U & $9: 14: 00$ & 103.432 & 103.544 & $\ldots{ }^{c}$ & $\ldots{ }^{c}$ & 2,5 & TTE/CTTE \\
\hline 18 & bn081003446 & $10: 42: 53$ & -0.038 & 0.090 & $120 \pm 14$ & $60 \pm 11$ & $1,2,5$ & TTE/CTTE \\
\hline 19 & bn081003779 & $18: 41: 39$ & -0.023 & 0.105 & $104 \pm 9$ & $60 \pm 11$ & $1,2,5$ & TTE/CTTE \\
\hline 20 & bn081004050 & $1: 11: 32$ & -0.023 & 0.105 & $132 \pm 28$ & $56 \pm 11$ & $1,2,5$ & TTE/CTTE \\
\hline 21 & bn081005020 & 0:29:09 & -0.023 & -0.007 & $24 \pm 16$ & $12 \pm 6$ & 6,7 & TTE/CTTE \\
\hline 22 & bn081010537 & $12: 53: 38$ & -0.039 & 0.009 & $60 \pm 18$ & $24 \pm 9$ & $1,2,5$ & TTE/CTTE \\
\hline 23 & bn090322789 & $18: 56: 23.75$ & -0.087 & 0.489 & $592 \pm 40$ & $360 \pm 29$ & $0,1,3$ & TTE/CTTE \\
\hline 24 & bn090322944 & $22: 39: 15.75$ & -0.023 & 0.489 & $288 \pm 6$ & $88 \pm 6$ & 7,8 & TTE/CTTE \\
\hline 25 & bn090326625 & $15: 00: 36.01$ & -0.023 & 0.089 & $244 \pm 30$ & $80 \pm 6$ & 9,10 & TTE/CTTE \\
\hline 26 & bn090326625U & $15: 00: 36.01$ & 2.176 & 2.496 & $192 \pm 45$ & $96 \pm 36$ & 9,10 & TTE/CTTE \\
\hline 27 & bn090328545 & $13: 05: 12.04$ & -0.023 & 0.057 & $136 \pm 37$ & $48 \pm 9$ & 9,10 & TTE/CTTE \\
\hline 28 & bn090329754 & $18: 05: 15.76$ & -0.023 & 0.025 & $140 \pm 54$ & $28 \pm 11$ & 10,11 & TTE/CTTE \\
\hline 29 & bn090330237 & 05:41:09.99 & -0.023 & 0.057 & $124 \pm 24$ & $32 \pm 9$ & 6,7 & TTE/CTTE \\
\hline 30 & bn090401093 & $02: 14: 28.53$ & -0.375 & -0.327 & $2656 \pm 172$ & $1632 \pm 172$ & $3,6,7$ & TTE/CTTE \\
\hline 31 & bn090401666 & $15: 59: 36.83$ & -0.023 & 0.89 & $88 \pm 6$ & $32 \pm 6$ & 10 & TTE/CTTE \\
\hline 32 & bn090403592 & $14: 13: 04.46$ & -0.023 & 0.121 & $320 \pm 23$ & $80 \pm 36$ & 9,10 & TTE/CTTE \\
\hline 33 & bn090403761 & $18: 15: 36.30$ & -0.039 & 0.041 & $136 \pm 18$ & $48 \pm 23$ & 6,7 & TTE/CTTE \\
\hline 34 & bn090409351 & $08: 25: 24.01$ & -0.023 & 0.073 & $112 \pm 18$ & $72 \pm 18$ & 9,10 & TTE/CTTE \\
\hline 35 & bn090411917 & $22: 01: 05.27$ & -0.039 & 0.137 & $180 \pm 20$ & $64 \pm 9$ & 7,8 & TTE/CTTE \\
\hline 36 & bn090413987 & $23: 41: 48.86$ & -0.007 & 0.057 & $72 \pm 6$ & $28 \pm 6$ & 6,7 & TTE/CTTE \\
\hline 37 & bn090417946 & $22: 42: 11.37$ & -0.023 & 0.409 & $480 \pm 69$ & $336 \pm 23$ & 9,10 & TTE/CTTE \\
\hline
\end{tabular}

Notes.

a yymmdd.thousandth of day.

${ }^{\mathrm{b}}$ Related to $T_{0}=$ trigger time.

c rmfit crashed.

in the second panel, reached a single detector peak count rate of about $>3.5 \times 10^{4}$ count s$^{-1}$, resulting in dead time of $10 \%$. The third panel shows the second brightest burst (burst 31 of Table 1) with an observed single detector peak count rate of $\sim 1 \times 10^{5}$ count $^{-1}$ and a dead time of $50 \%$. The nonsaturated bursts of the 2009 March-April period are on average brighter than the bursts observed in 2008 October. The rightmost panel of the bottom row shows burst 25 , which is comparable in brightness to the brightest burst observed during 2008 October.

Table 1 lists detailed trigger information for all events as well as the time periods (start and stop columns) used for our spectral analysis. The high temporal resolution of the TTE $(2 \mu \mathrm{s})$ easily accommodates the analysis of the bursts. The $0.064 \mathrm{~s}$ continuous CTIME data cannot be used for our spectral analysis due to their coarser spectral resolution. CSPEC data were used only for burst 15 (Table 1), which was located inside the one CSPEC $1.024 \mathrm{~s}$ integration time with a signal to background ratio high enough to allow spectral analysis (this burst was excluded from our temporal analysis).

\subsection{Soft Burst Location Systematics}

We determined for all 21 triggered bursts in Table 1 an approximate on-ground location using the TRIGDAT data in order to check the consistency of the GBM derived locations with the accurately known source position. These locations were computed using the Daughter Of Locburst (DOL) location-finding code, which compares observed rates to a table of calculated relative rates in the $12 \mathrm{NaI}$ detectors for each of 41,168 directions (at $1^{\circ}$ resolution) in spacecraft coordinates by utilizing a chi-squared fit. Compared to the on-board flight software locations, the DOL includes atmospheric and spacecraft scattering more accurately, using a finer angular grid, and accounts more properly for differences in burst spectra. In addition, DOL also corrects for the recording dead time. A dedicated table was prepared especially for soft events with rates integrated over the 5-50 keV energy range.

The right panel of Figure 4 shows the calculated locations and their statistical uncertainties (radius of $1 \sigma$ containment) for both 


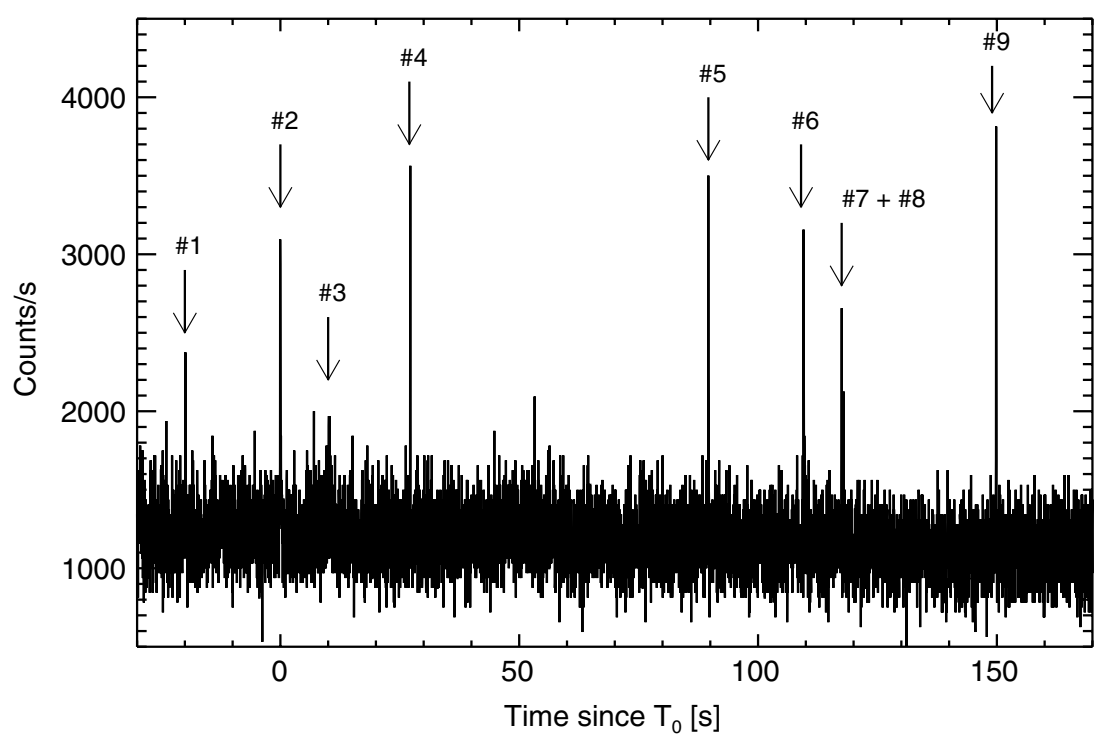

Figure 2. First GBM trigger (bn081003.377) from SGR J1550-5418, on 2008 October 3, at 09:03:06 UT (244717387 MET). Besides the triggering event (no. 2), eight additional, untriggered, bursts (nos. 1,3-9) are also visible (marked with arrows), including burst 1 observed prior to the trigger time $\left(T_{0} \sim 0 \mathrm{~s}\right)$ at $-19.9 \mathrm{~s}$.
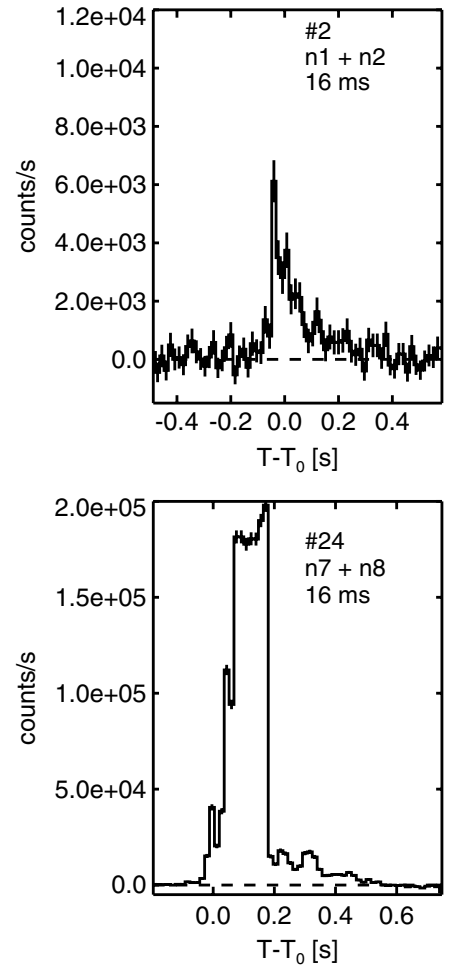
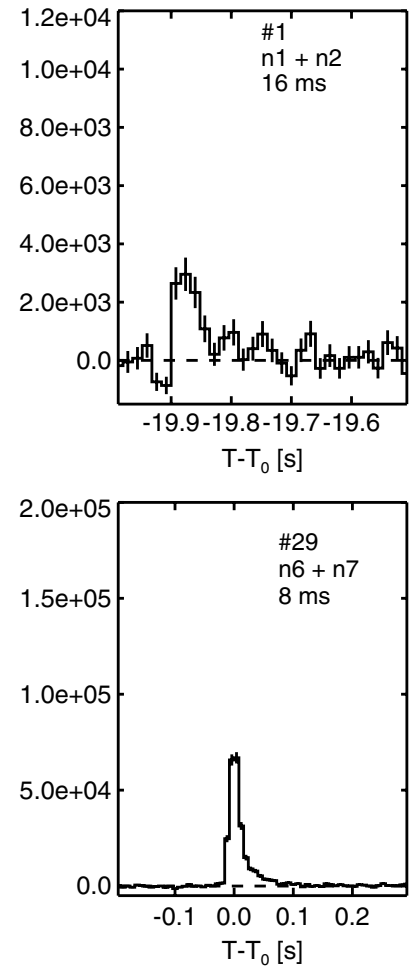
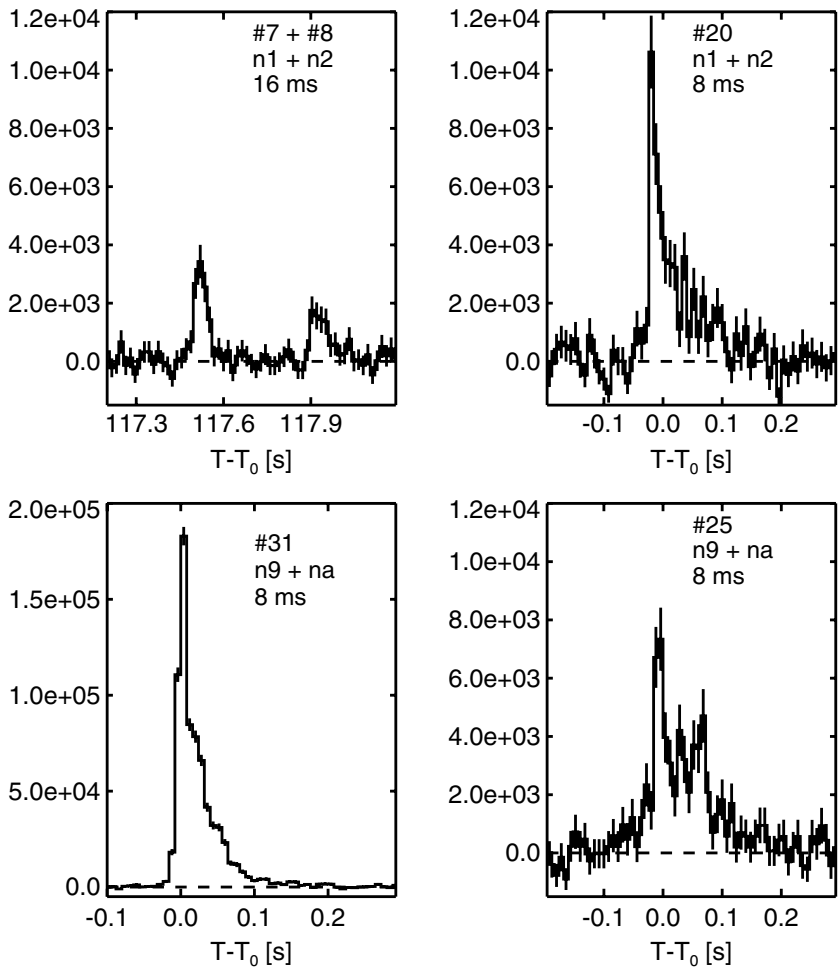

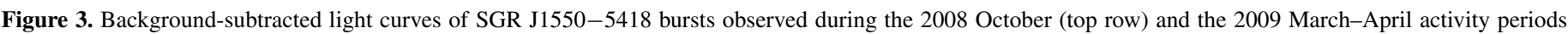

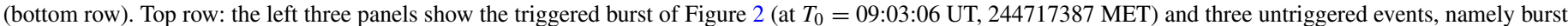

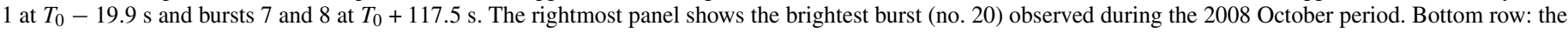

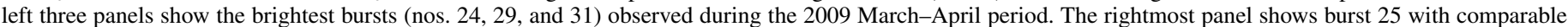

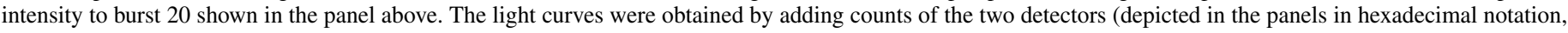
$\mathrm{n} 0 . . \mathrm{nb})$, which showed the highest peak count rates.

activity periods. The two left panels show the location accuracy distributions as absolute distance in degrees from the source position (top) and as distance in number of $1 \sigma$ radii (bottom). Roughly $86 \%$ of the locations are consistent with the source position within $3 \sigma$ error radii. A similar percentage is located $<8^{\circ}$ from the actual source position in a flat distribution. It should be noted that the three bursts (nos. 31, 45, and 36) with the largest deviation of error radii $(>3)$, are the ones with the highest $16 \mathrm{~ms}$ peak fluxes, listed in Table 2, with the exception of trigger bn090322.944, the saturated burst 24 (see Figure 3, bottom row, leftmost panel). In this case, the location was derived for one selected $\sim 50 \mathrm{~ms}$ bin in the rising part of the burst light curve, from $-0.048 \mathrm{~s}$ to $0.016 \mathrm{~s}$, thus avoiding the saturated part. From these dependencies, we conclude that the "soft" location accuracy is affected by high event rates, probably caused by hidden systematic errors, e.g., the standard dead-time correction may not be valid for soft and bright events.

The accuracy of the GBM localizations for (the hard) GammaRay Bursts (GRBs) is reported by V. Connaughton et al. (2012, in preparation). They find that the systematic location errors for 

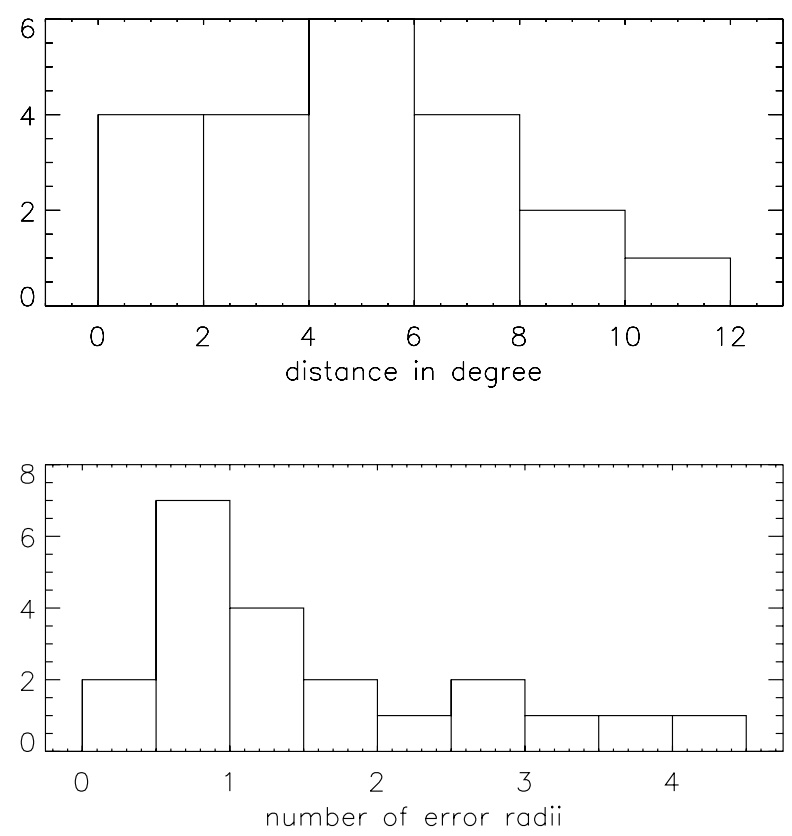

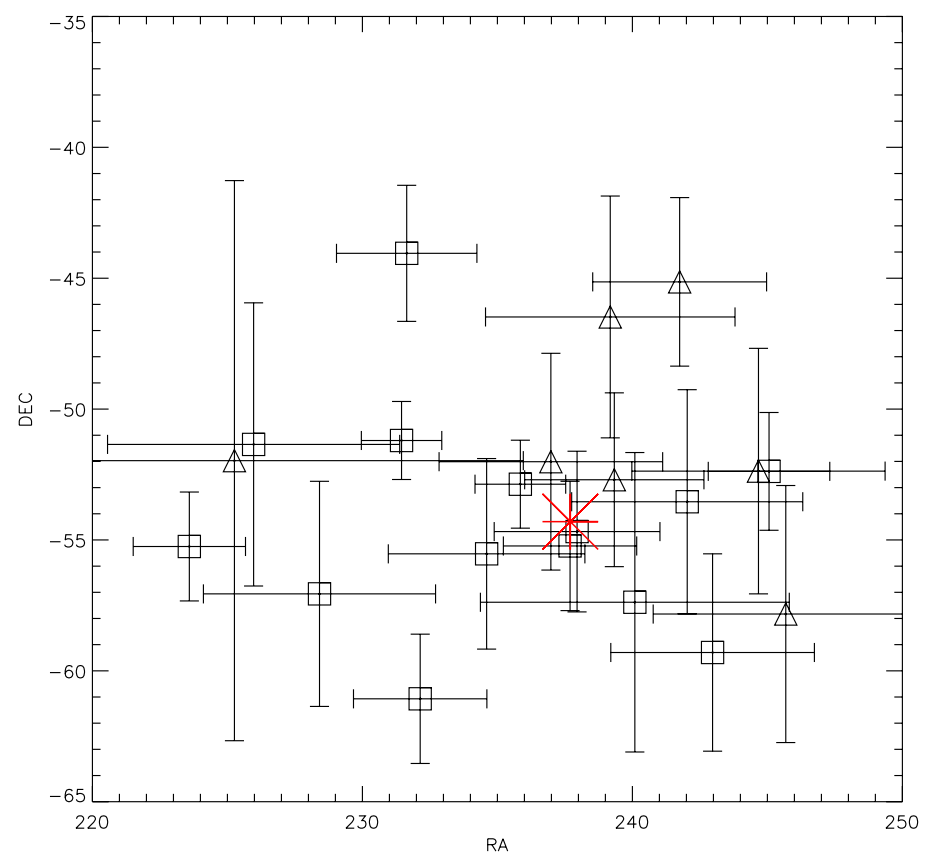

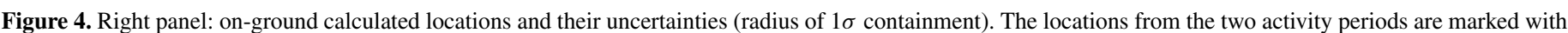

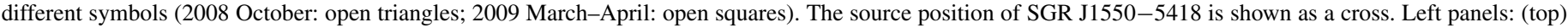
location accuracy, displayed as absolute distance in degrees from the source position and (bottom) as distance in number of error radii.

(A color version of this figure is available in the online journal.)

GRBs are best described by two contributions, one with 2.8 error with $70 \%$ weight and one with 8.4 error with $30 \%$ weight.

\subsection{Temporal Analysis \\ 3.3.1. $T_{90}$ and $T_{50}$ Durations}

The SGR J1550-5418 burst temporal analysis was performed in a similar manner as the one described by Lin et al. (2011). The durations, expressed as $T_{90}$ and $T_{50},{ }^{11}$ were determined both in photon and in count space. The durations in count space were calculated by applying an algorithm adapted for the analysis of GBM TTE data, which was originally developed by Kouveliotou et al. (1993) and later modified by Göğüş et al. (2001) and Gavriil et al. (2004). Thanks to a new on-ground build data type of the GBM data, the so-called CTTE data type, it was possible to determine the $T_{90}, T_{50}$ durations in photon space using the GBM RMFIT (4.0rc01) software, ${ }^{12}$ similar to what was done in the case of GBM GRBs (Paciesas et al. 2012). The CTTE data type is derived from TTE data by rebinning the 128 TTE energy bins into eight energy bins, with the same boundaries as the CTIME data. The CTTE data type allows us to generate finer time bins, compared to the $64 \mathrm{~ms}$ bin width of the CTIME data during burst mode, which are necessary especially for temporal analysis of short bursts from SGRs. In our case, the individual burst data were rebinned to $4 \mathrm{~ms}, 8 \mathrm{~ms}$, or $16 \mathrm{~ms}$ bins depending on the intensity of the burst. The burst durations $T_{90}\left(T_{50}\right)$ were computed in the 8-200 keV energy range.

Since the bursts presented here are relatively weak, we applied the count rate algorithm twice to each burst in order to account for systematics. In some cases, the deviations were large, most

\footnotetext{
$11 T_{90}\left(T_{50}\right)$ is the duration during which the background-subtracted cumulative counts increase from 5\% (25\%) to $95 \%(75 \%)$ of the total counts (Kouveliotou et al. 1993).

12 R. S. Mallozzi, R. D. Preece, \& M. S. Briggs, "RMFIT, A Lightcurve and Spectral Analysis Tool," (c) 2008, Robert D. Preece, University of Alabama in Huntsville, 2008.
}

probably depending on small differences in the background selection. Durations calculated in photon space have smaller systematic uncertainties. Due to this fact, only the results obtained in photon space are presented here.

Figure 5 shows the distribution of $T_{90}$ and $T_{50}$ durations for 30 bursts out of the 37 bursts listed in Table 1 . The mean value of $\sim 170 \mathrm{~ms}$ of the log-normal fit to the $T_{90}$ distribution is comparable to the bulk of magnetar bursts and entirely consistent with the average duration of $174 \pm 10 \mathrm{~ms}$ of the bursts during the most active episode from the source (2009 January) reported by van der Horst et al. (2012). No general trends or differences in the burst durations of the two active periods were observed.

\subsubsection{Timing Analysis}

In 2008 October, SGR J1550-5418 emitted 19 bursts in the course of $\sim 10 \mathrm{hr}$. Out of these bursts, 15 events were emitted within only $\sim 10$ minutes. Similarly to Kaneko et al. (2010), we looked for periodic modulations in the source persistent emission using the TTE data stream of NaI 1, which was the detector with the smallest source angle toward SGR J1550-5418. For the analysis, we corrected the TTE data to the solar system barycenter, binned the data with a time resolution of $16 \mathrm{~ms}(8-100 \mathrm{keV})$, and selected the time interval $\left[T_{0}-25 ; T_{0}+160\right] \mathrm{s}$ which roughly corresponds to the interval from burst 1 to 9 (see Table 1). We performed all timing analyses twice, with the SGR bursts and without them (we carefully removed the burst intervals from the light curve).

We used several different timing techniques, including a Lomb-Scargle periodogram (Scargle 1982; Horne \& Baliunas 1986; Press \& Rybciki 1989), Period04 ${ }^{13}$ (Lenz \& Breger 2005), a code specifically designed to extract multiple periodic signals in astronomical time series through simultaneous least-squares fitting. We also used a multi-harmonic "analysis of variance"

\footnotetext{
13 http://www.univie.ac.at/tops/Period04/
} 


\begin{tabular}{|c|c|c|c|c|c|c|c|c|c|c|c|c|c|}
\hline \multirow{2}{*}{$\begin{array}{l}\text { Burst } \\
\text { No. }\end{array}$} & \multicolumn{3}{|c|}{$\mathrm{BB}$} & \multicolumn{3}{|c|}{ OTTB } & \multicolumn{4}{|c|}{ Comp } & \multirow{2}{*}{$\begin{array}{c}\Delta C \text {-stat } \\
(\mathrm{BB} \\
- \text { OTTB })\end{array}$} & \multirow{2}{*}{$\begin{array}{c}\text { Fluence } \\
(8-200 \mathrm{keV}) \\
\left(10^{-8} \mathrm{erg} \mathrm{cm}^{-2}\right)\end{array}$} & \multirow{2}{*}{$\begin{array}{c}16 \text { ms Peak Flux } \\
(8-200 \mathrm{keV}) \\
\left(\text { photon } \mathrm{cm}^{-2} \mathrm{~s}^{-1}\right)\end{array}$} \\
\hline & $\begin{array}{c}A \\
\times 10^{-3}\end{array}$ & $k T$ & $\begin{array}{l}C \text {-stat } \\
/ \text { DOF }\end{array}$ & $\begin{array}{c}A \\
\times 10^{-3}\end{array}$ & $k T$ & $\begin{array}{l}C \text {-stat } \\
/ \mathrm{DOF}\end{array}$ & $A$ & $\begin{array}{l}E_{\text {Peak }} \\
(\mathrm{keV})\end{array}$ & $\alpha$ & $\begin{array}{l}C \text {-stat } \\
/ \text { DOF }\end{array}$ & & & \\
\hline 1 & $4.7_{-1.3}^{+1.8}$ & $11.5_{-1.0}^{+1.0}$ & $165.3 / 237$ & $16.8_{-4.0}^{+4.9}$ & $44.7_{-6.6}^{+9.1}$ & $169.5 / 237$ & $\ldots$ & $\ldots$ & $\ldots$ & $\ldots$ & -4.2 & $4.1 \pm 0.5$ & $14.6 \pm 3.0$ \\
\hline 2 & $3.1_{-0.5}^{+0.5}$ & $12.5_{-0.5}^{+0.6}$ & $215.3 / 237$ & $17.1_{-1.9}^{+2.8}$ & $52.5_{-4.2}^{+7.2}$ & $275.5 / 237$ & $\ldots$ & $\ldots$ & $\ldots$ & $\ldots$ & -60.2 & $13.4 \pm 0.9$ & $23.0 \pm 3.4$ \\
\hline 3 & $1.2_{-0.3}^{+0.5}$ & $11.4_{-1.0}^{+1.0}$ & $264.2 / 237$ & $4.8_{-1.2}^{+1.4}$ & $49.5_{-8.4}^{+11.2}$ & $270.8 / 237$ & $\ldots$ & $\ldots$ & $\ldots$ & $\ldots$ & -6.6 & $8.1 \pm 0.9$ & $9.9 \pm 2.6$ \\
\hline 4 & $2.5_{-0.6}^{+0.8}$ & $12.8_{-0.9}^{+1.0}$ & $194.1 / 237$ & $17.6_{-3.1}^{+3.1}$ & $59.5_{-8.8}^{+10.4}$ & $210.6 / 237$ & $\ldots$ & $\ldots$ & $\ldots$ & $\ldots$ & -16.6 & $6.5 \pm 0.6$ & $23.1 \pm 3.3$ \\
\hline 5 & $2.6_{-0.5}^{+0.7}$ & $13.1_{-0.8}^{+0.8}$ & $193.7 / 237$ & $18.4_{-2.9}^{+3.1}$ & $56.0_{-6.7}^{+8.4}$ & $226.1 / 237$ & $\ldots$ & $\ldots$ & $\ldots$ & $\ldots$ & -32.4 & $7.6 \pm 0.7$ & $26.3 \pm 3.5$ \\
\hline 6 & $2.3_{-0.4}^{+0.5}$ & $12.3_{-0.6}^{+0.7}$ & $221.2 / 237$ & $12.2_{-1.7}^{+2.1}$ & $52.5_{-5.4}^{+7.5}$ & $256.1 / 237$ & $\ldots$ & $\ldots$ & $\ldots$ & $\ldots$ & -35.0 & $13.1 \pm 1.0$ & $21.7 \pm 3.4$ \\
\hline 7 & $4.0_{-1.1}^{+1.6}$ & $10.8_{-0.9}^{+1.0}$ & $218.4 / 237$ & $14.8_{-3.6}^{+3.9}$ & $54.5_{-10.0}^{+13.4}$ & $226.7 / 237$ & $\ldots$ & $\ldots$ & $\ldots$ & $\ldots$ & -8.3 & $4.5 \pm 0.5$ & $18.6 \pm 3.2$ \\
\hline 8 & $0.7_{-0.3}^{+0.5}$ & $13.6_{-1.8}^{+2.1}$ & $230.7 / 237$ & $8.0_{-2.4}^{+2.4}$ & $70.3_{-0.0}^{+30.5}$ & $228.4 / 237$ & $\ldots$ & $\ldots$ & $\ldots$ & $\ldots$ & +2.3 & $3.6 \pm 0.6$ & $8.3 \pm 2.4$ \\
\hline 9 & $3.2_{-0.5}^{+0.6}$ & $13.2_{-0.6}^{+0.6}$ & $211.2 / 237$ & $25.2_{-3.0}^{+3.4}$ & $59.0_{-5.9}^{+7.5}$ & $237.3 / 237$ & $\ldots$ & $\ldots$ & $\ldots$ & $\ldots$ & -26.1 & $1.3 \pm 0.1$ & $26.5 \pm 3.6$ \\
\hline 10 & $4.7_{-1.5}^{+2.1}$ & $11.1_{-1.0}^{+1.2}$ & $159.1 / 237$ & $13.2_{-3.9}^{+7.2}$ & $42.9_{-0.0}^{+17.1}$ & $172.3 / 237$ & $\ldots$ & $\ldots$ & $\ldots$ & $\ldots$ & -13.2 & $2.3 \pm 0.4$ & $22.6 \pm 3.5$ \\
\hline $1-10$ & $2.3_{-0.2}^{+0.2}$ & $12.3_{-0.3}^{+0.3}$ & $237.5 / 237$ & $12.8_{-0.8}^{+0.8}$ & $54.2_{-2.5}^{+2.9}$ & $402.7 / 237$ & $10.9_{-5.6}^{+5.6}$ & $47.7_{-1.1}^{+1.1}$ & $1.51_{-0.26}^{+0.27}$ & $238.3 / 236$ & -165.2 & $\ldots$ & $\ldots$ \\
\hline 15 & $5.1_{-0.2}^{+0.2}$ & $12.0_{-1.1}^{+1.2}$ & $356.7 / 359$ & $3.0_{-0.6}^{+0.6}$ & $56.2_{-9.3}^{+11.3}$ & $354.5 / 359$ & $0.19_{-0.17}^{+0.33}$ & $49.9_{-5.7}^{+12.6}$ & $0.25_{-1.11}^{+0.47}$ & $352.7 / 358$ & +2.2 & $11.2 \pm 0.9$ & $\ldots$ \\
\hline 16 & $1.2_{-0.3}^{+0.4}$ & $12.5_{-0.9}^{+1.0}$ & $338.6 / 340$ & $6.3_{-1.3}^{+1.8}$ & $50.5_{-6.7}^{+11.8}$ & $359.9 / 340$ & $86.5_{-53.0}^{+2160}$ & $47.4_{-3.2}^{+3.2}$ & $2.91_{-0.96}^{+1.84}$ & $336.9 / 339$ & -21.3 & $5.4 \pm 0.6$ & $18.0 \pm 2.9$ \\
\hline 17 & $4.4_{-0.7}^{+0.8}$ & $12.6_{-0.6}^{+0.6}$ & $187.6 / 228$ & $26.2_{-3.5}^{+3.3}$ & $55.7_{-5.8}^{+6.0}$ & $218.2 / 228$ & $6.3_{-3.3}^{+32.8}$ & $49.5_{-3.4}^{+3.9}$ & $0.84_{-0.35}^{+0.97}$ & $187.5 / 227$ & -30.6 & $12.7 \pm 0.6$ & $28.6 \pm 2.8$ \\
\hline 18 & $5.4_{-0.7}^{+0.9}$ & $12.5_{-0.5}^{+0.5}$ & $329.4 / 347$ & $30.1_{-3.1}^{+3.1}$ & $51.0_{-4.0}^{+4.1}$ & $348.5 / 347$ & $4.1_{-2.1}^{+4.8}$ & $49.4_{-2.2}^{+2.4}$ & $0.53_{-0.36}^{+0.40}$ & $324.8 / 346$ & -19.1 & $17.3 \pm 0.7$ & $39.1 \pm 3.0$ \\
\hline 19 & $8.8_{-0.1}^{+0.1}$ & $10.7_{-0.4}^{+0.4}$ & $383.7 / 358$ & $22.8_{-2.7}^{+2.7}$ & $42.9_{-3.1}^{+3.3}$ & $392.8 / 358$ & $3.12_{-1.45}^{+3.03}$ & $42.4_{-1.8}^{+2.0}$ & $0.26_{-0.31}^{+0.33}$ & $371.5 / 357$ & -9.1 & $15.0 \pm 0.5$ & $44.8 \pm 2.7$ \\
\hline 20 & $4.0_{-0.5}^{+0.6}$ & $12.6_{-0.5}^{+0.5}$ & $327.1 / 360$ & $24.9_{-2.6}^{+2.7}$ & $55.7_{-4.7}^{+5.3}$ & $369.3 / 360$ & $9.6_{-5.2}^{+13.3}$ & $49.2_{-2.1}^{+2.3}$ & $1.14_{-0.40}^{+0.44}$ & $326.5 / 359$ & -42.2 & $13.0 \pm 0.5$ & $51.7 \pm 4.0$ \\
\hline 21 & $1.1_{-0.3}^{+0.4}$ & $11.9_{-1.0}^{+1.0}$ & $143.1 / 241$ & $52.1_{-11.2}^{+11.8}$ & $49.3_{-7.4}^{+8.9}$ & $154.6 / 241$ & $4.9_{-3.3}^{+15.4}$ & $47.9_{-4.4}^{+5.3}$ & $0.3_{-0.6}^{+0.7}$ & $140.4 / 240$ & -11.5 & $3.8 \pm 0.3$ & $43.05 \pm 3.1$ \\
\hline 22 & $2.6_{-0.6}^{+0.7}$ & $14.0_{-0.9}^{+1.0}$ & $305.3 / 358$ & $26.4_{-4.1}^{+4.2}$ & $61.5_{-8.4}^{+10.1}$ & $328.5 / 358$ & $20.4_{-14.5}^{+105.0}$ & $53.9_{-3.5}^{+3.9}$ & $1.7_{-0.7}^{+1.0}$ & $305.4 / 357$ & -23.2 & $5.0 \pm 0.3$ & $20.35 \pm 1.9$ \\
\hline 23 & $3.5_{-0.6}^{+0.9}$ & $10.1_{-0.6}^{+0.5}$ & $562.8 / 351$ & $9.5_{-1.1}^{+1.1}$ & $47.9_{-3.8}^{+4.2}$ & $442.7 / 351$ & $0.02_{-0.01}^{+0.01}$ & $43.3_{-8.1}^{+8.7}$ & $-1.63_{-0.16}^{+0.18}$ & $431.31 / 350$ & +120.1 & $30.9 \pm 1.6$ & $34.3 .0 \pm 2.7$ \\
\hline 25 & $12.3_{-2.5}^{+3.2}$ & $9.4_{-0.6}^{+0.6}$ & $268.8 / 231$ & $18.7_{-3.5}^{+3.1}$ & $40.0_{-4.2}^{+4.8}$ & $232.7 / 231$ & $183_{-83}^{+177}$ & $39.7_{-4.9}^{+5.1}$ & $-1.1_{-0.8}^{+1.1}$ & $232.6 / 230$ & +36.1 & $13.2 \pm 0.9$ & $55.0 \pm 5.9$ \\
\hline 26 & $0.5_{-0.2}^{+0.3}$ & $13.8_{-1.9}^{+2.0}$ & $299.2 / 254$ & $7.6_{-1.6}^{+1.5}$ & $105.0_{-30.0}^{+48.8}$ & $298.2 / 254$ & $0.10 \pm 0.14$ & $69.7 \pm 15.8$ & $-0.18 \pm 0.78$ & $297.11 / 253$ & +1.0 & $9.5 \pm 3.3$ & $7.7 \pm 1.1$ \\
\hline 27 & $16.7_{-3.0}^{+3.8}$ & $9.4_{-0.5}^{+0.5}$ & $202.9 / 235$ & $23.8_{-4.3}^{+4.7}$ & $39.0_{-3.8}^{+4.3}$ & $177.6 / 235$ & $0.64_{-0.32}^{+0.73}$ & $39.2_{-3.2}^{+3.6}$ & $-0.66_{-0.32}^{+0.35}$ & $176.4 / 234$ & +25.3 & $12.6 \pm 0.6$ & $53.3 \pm 4.2$ \\
\hline 28 & $29.3_{-5.2}^{+6.9}$ & $9.4_{-0.5}^{+0.5}$ & $191.9 / 238$ & $35.2_{-6.6}^{+7.3}$ & $35.6_{-3.4}^{+3.8}$ & $173.1 / 238$ & $1.47_{-0.8}^{+2.0}$ & $36.8_{-3.1}^{+3.2}$ & $-0.56_{-0.36}^{+0.40}$ & $171.6 / 237$ & +18.8 & $12.7 \pm 0.6$ & $97.7 \pm 6.3$ \\
\hline 29 & $97.4_{-7.2}^{+8.5}$ & $8.9_{-0.2}^{+0.2}$ & $417.9 / 239$ & $79.7_{-6.5}^{+6.8}$ & $32.6_{-1.2}^{+1.3}$ & $226.2 / 239$ & $2.23_{-0.56}^{+0.77}$ & $33.3_{-1.4}^{+1.3}$ & $-0.87_{-1.3}^{+1.4}$ & $225.3 / 238$ & +191.7 & $55.7 \pm 1.0$ & $518 \pm 12$ \\
\hline 30 & $3.0_{-1.3}^{+2.4}$ & $10.7_{-1.6}^{+1.7}$ & $278.0 / 360$ & $10.3_{-4.0}^{+4.4}$ & $49.4_{-13.1}^{+19.7}$ & $271.2 / 362$ & $0.01 \pm 0.05$ & $76.7 \pm 114.0$ & $-1.84 \pm 0.43$ & $270.0 / 359$ & +6.8 & $2.5 \pm 0.3$ & $14.1 \pm 1.7$ \\
\hline 31 & $261_{-13}^{+14}$ & $8.8_{-0.1}^{+0.1}$ & $361.3 / 118$ & $176_{-11}^{+11}$ & $30.9_{-0.8}^{+0.8}$ & $158.8 / 118$ & $17.9_{-3.7}^{+4.7}$ & $33.2_{-0.7}^{+0.7}$ & $-0.36_{-0.10}^{+0.11}$ & $117.0 / 117$ & +202.5 & $186.7 \pm 2.4$ & $1122 \pm 26$ \\
\hline 32 & $24.3_{-3.4}^{+4.3}$ & $8.5_{-0.4}^{+0.4}$ & $309.3 / 237$ & $18.4_{-2.8}^{+3.0}$ & $34.1_{-2.4}^{+2.7}$ & $247.5 / 237$ & $0.37_{-0.15}^{+0.26}$ & $34.2_{-2.7}^{+2.7}$ & $-0.96_{-0.23}^{+0.24}$ & $247.5 / 236$ & +61.8 & $21.4+/ 0.7$ & $92.6 \pm 5.1$ \\
\hline 33 & $9.8_{-2.4}^{+3.6}$ & $9.5_{-0.8}^{+0.8}$ & $246.9 / 241$ & $19.1_{-4.0}^{+4.4}$ & $44.7_{-5.9}^{+7.2}$ & $218.1 / 241$ & $0.08_{-0.04}^{+0.09}$ & $45.6_{-8.6}^{+10.8}$ & $-1.38_{-0.0}^{+0.35}$ & $217.0 / 240$ & +28.8 & $8.4 \pm 0.5$ & $42.5 \pm 3.5$ \\
\hline 34 & $54.0_{-6.8}^{+8.1}$ & $7.9_{-0.3}^{+0.3}$ & $251.0 / 237$ & $22.0_{-3.5}^{+3.9}$ & $29.0_{-1.8}^{+2.0}$ & $216.1 / 237$ & $1.54_{-0.63}^{+2.33}$ & $30.5_{-2.1}^{+2.1}$ & $-0.63_{-0.25}^{+0.40}$ & $213.7 / 236$ & +34.9 & $23.3 \pm 0.7$ & $149 \pm 7$ \\
\hline 35 & $41.1_{-4.0}^{+4.8}$ & $8.5_{-0.2}^{+0.2}$ & $332.9 / 241$ & $28.2_{-2.9}^{+3.1}$ & $31.8_{-1.5}^{+1.5}$ & $217.5 / 241$ & $0.97_{-0.30}^{+0.45}$ & $32.6_{-1.6}^{+1.5}$ & $-0.82_{-0.17}^{+0.17}$ & $216.2 / 240$ & +115.4 & $45.5 \pm 1.0$ & $258 \pm 9$ \\
\hline 36 & $60.2_{-5.1}^{+5.8}$ & $9.7_{-0.2}^{+0.2}$ & $295.0 / 240$ & $77.6_{-7.0}^{+7.4}$ & $35.4_{-1.6}^{+1.7}$ & $213.4 / 240$ & $3.7_{-1.1}^{+1.8}$ & $37.1_{-1.4}^{+1.4}$ & $-0.50_{-0.17}^{+0.18}$ & $204.6 / 239$ & +81.6 & $37.7 \pm 0.8$ & $364 \pm 10$ \\
\hline 37 & $5.3_{-1.1}^{+1.6}$ & $9.3_{-0.6}^{+0.6}$ & $331.0 / 237$ & $9.5_{-1.5}^{+1.7}$ & $44.5_{-4.6}^{+5.4}$ & $280.6 / 237$ & $0.03_{-0.01}^{+0.02}$ & $45.2_{-7.8}^{+8.8}$ & $-1.49_{-0.0}^{+0.25}$ & $277.2 / 236$ & +51.0 & $22.7 \pm 1.0$ & $64.7 \pm 4.6$ \\
\hline
\end{tabular}



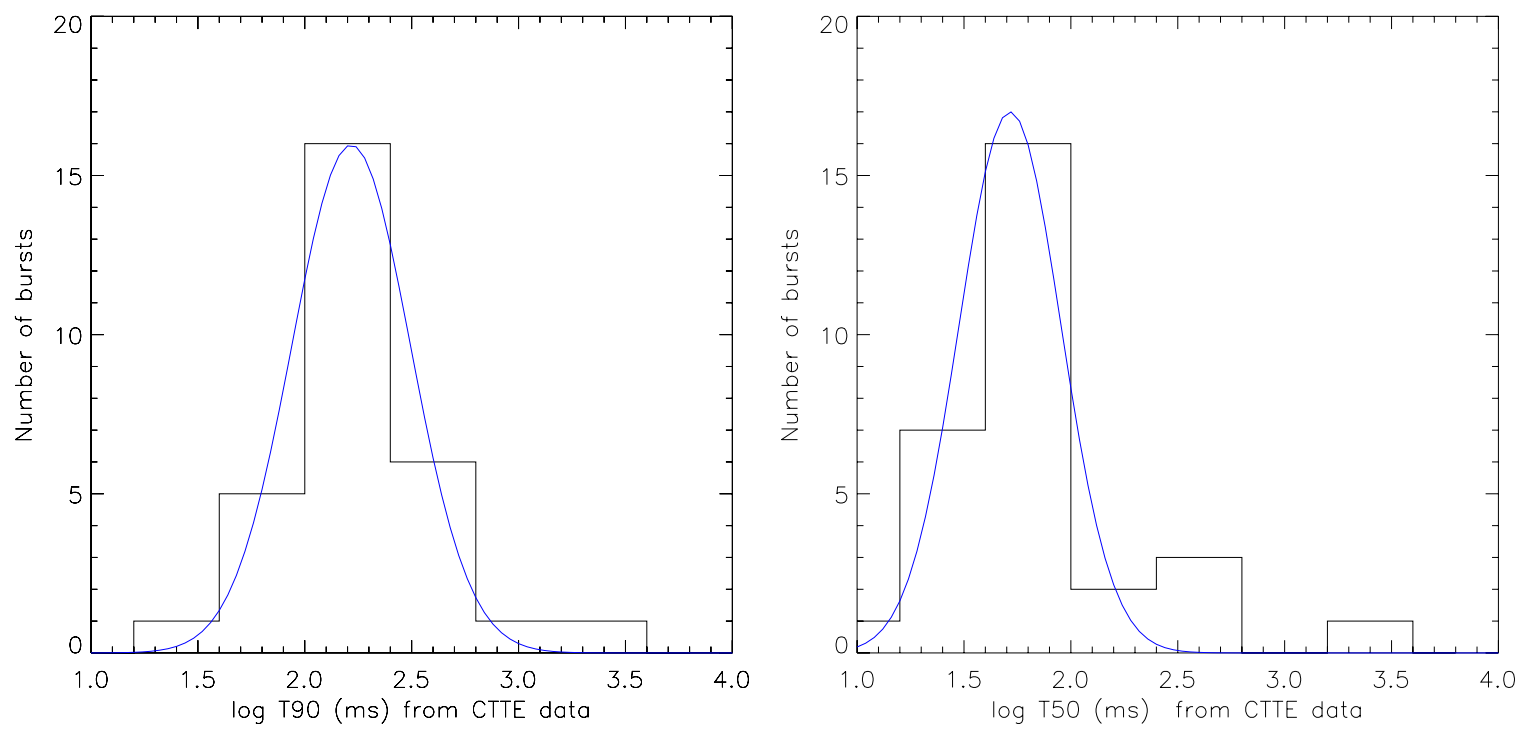

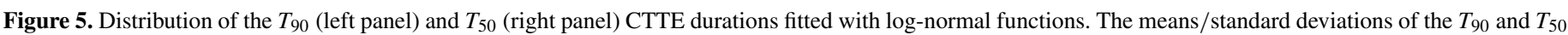

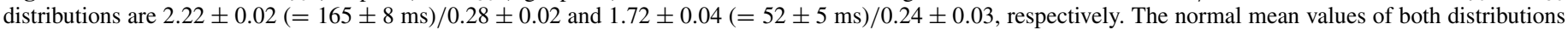
are $278 \mathrm{~ms}$ and $138 \mathrm{~ms}$, respectively.

(A color version of this figure is available in the online journal.)

periodogram (mhAoV) of Schwarzenberg-Czerny (1996), and the method introduced by Vaughan (2005). After correcting for the number of trials, no significant frequency was found in either of the data sets.

An intriguing finding was in the burst arrival times, namely the time separation between bursts. Starting from the first event, 8 out of 16 bursts occur with a time separation in multiples of $10 \pm 0.5 \mathrm{~s}$. It is established observationally that SGR bursts occur randomly distributed in rotational phase (Mereghetti 2008). We performed a simple Monte Carlo simulation to test the significance of this result. Our simulations showed that the observed burst interval corresponds to a single-trial probability of $P=6.1 \times 10^{-5}$, i.e., a significance of $\sim 4 \sigma$. It is hard to accurately quantify a number of trials a posteriori. Considering the fact that there are various SGR/AXP sources, with several multiple prolific bursting episodes, the probability will be in general smaller. Since the January emission was heavily concentrated in a $24 \mathrm{hr}$ period with a very large number of bursts, we have not performed a detailed search for the $10 \mathrm{~s}$ period during this epoch. Only future observations will reveal whether this periodicity is indeed real and related specifically to SGR J1550-5418, or simply an observational artifact.

\subsection{Spectral Analysis}

We fit all bursts in Table 1 with the following models, which are known to best approximate SGR spectra: a simple PL function, a PL function with an exponential high-energy cutoff (Compt), a BB and an optically thin thermal bremsstrahlung (OTTB) spectrum, and a combined BB + BB model. We used the RMFIT (4.0rc01) spectral analysis software developed for the GBM data analysis. Table 2 lists the parameters of all single models (with the exception of the PL model) for both activity periods.

Combining all our fit results we find that the 2008 October burst spectra are best fitted with a single BB model. This is corroborated by the fact that the brightest burst observed during that period (burst 20 of Table 1) as well as a stacked spectrum derived from all bursts observed during the TTE data period of the bn081003.377 trigger (burst 1-10 of Table 1) are best fitted by the BB model as compared to fits with the OTTB and PL models. The BB and OTTB fits to the stacked spectrum are shown in Figure 6. We note that the residuals of the OTTB fit show a systematic wiggle, causing a $\Delta C$-stat between the two models of $\sim 165$. The same deviations were observed for burst 20 , this time with a $\Delta C$-stat of $\sim 40$. A fit with the Compt model yields in both cases a $C$-stat comparable to the value obtained for the BB fit, but the Compt model has one degree of freedom (DOF) less. For most of the bursts during the 2008 October period, the $\Delta C$-stat for the $\mathrm{BB}$ model was $>6$ with the exception of some weak bursts, which were also well fitted by an OTTB or PL model. A fit with a normal function to the distribution of single BB temperature $k T$ yields a mean value of $12.4 \pm 0.2 \mathrm{keV}$ (width $0.9 \pm 0.1 \mathrm{keV}$ ). The preference for a single BB model as best-fit model was also reported by Israel et al. (2010) for bursts observed during 2008 October with Swift-BAT in the 15-100 keV energy range. These bursts are all well described by a single BB function with temperatures $\sim 10 \mathrm{keV}$.

We also tried to fit the 2008 October bursts, even though they are faint, with the combined two BB functions, since Lin et al. (2012) reported that broadband (0.5-200 keV) SwiftXRT/GBM spectral fits show, on average, that the burst spectra are better described with the two BB functions than with the Comptonized model. As was expected, these fits were not able to constrain simultaneously the parameters of both $\mathrm{BB}$ components, even when fixing the ratio of the fluence in the two $\mathrm{BB}$ components, so that the hot component has twice the flux of the cold component, which is the mean ratio with relatively modest scatter that is found in Lin et al. (2012).

In contrast, the bursts from the 2009 March-April period are best fitted in almost all cases by an OTTB model. In order to investigate whether this difference is connected with the burst brightness difference between the two periods, we selected a burst with comparable brightness from each period, as shown in the rightmost panels of Figure 3, the brightest of the 2008 October activity period, already presented above (burst 20 of Table 1), as well as a burst among the fainter ones of the 2009 period (burst 25 of Table 1). The results of the BB, OTTB and Comp model fits for burst 25 are listed in Table 2, 

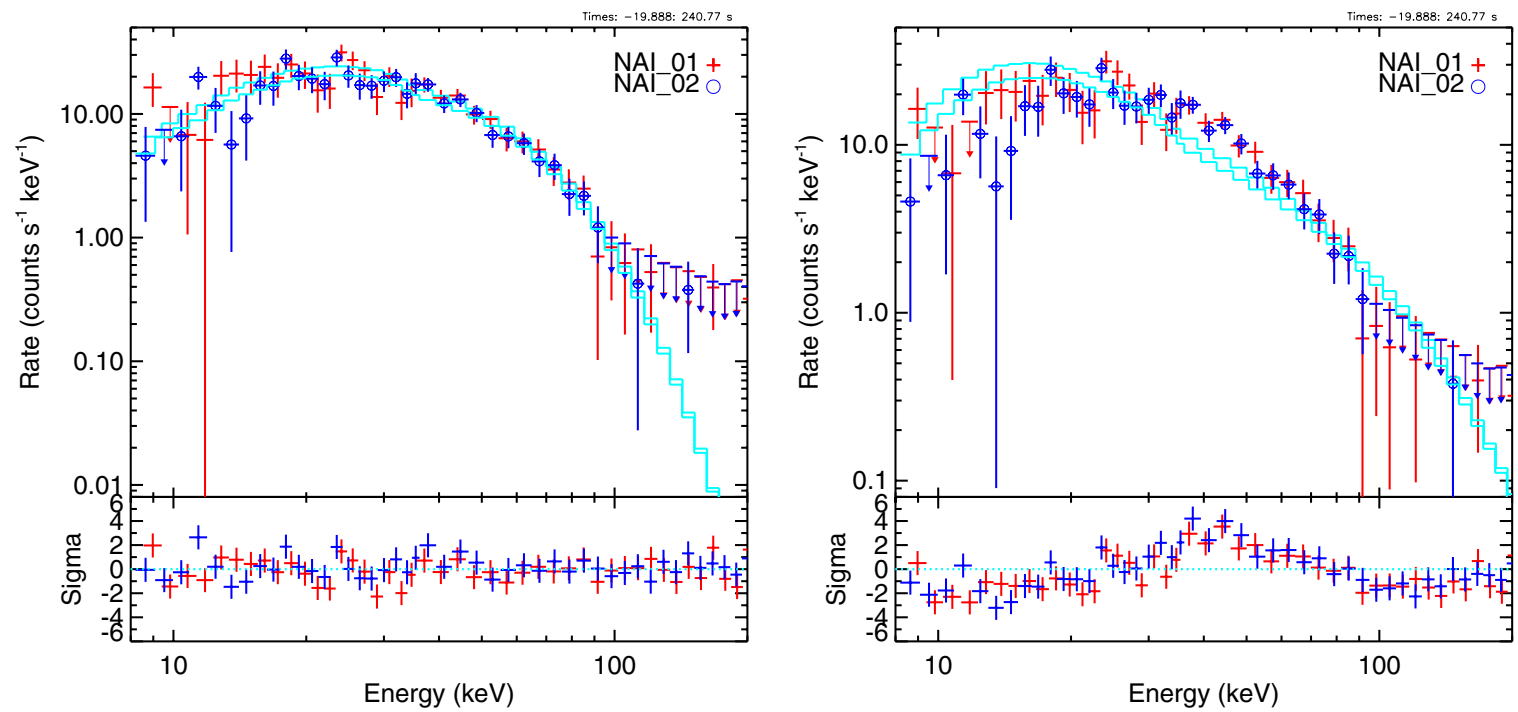

Figure 6. Stacked spectra of bn0810030.377. Left: BB fit; right: OTTB fit.

(A color version of this figure is available in the online journal.)
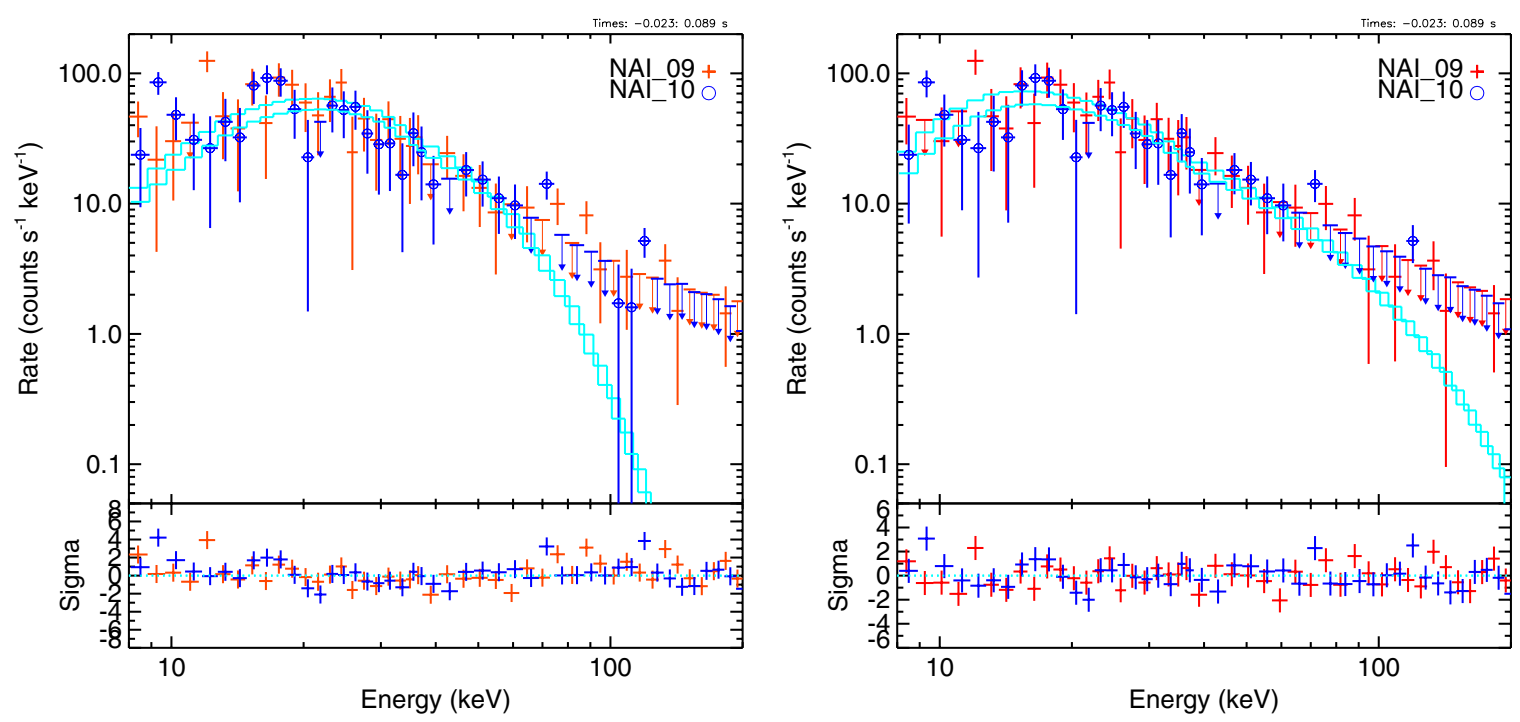

Figure 7. Spectra of bn090326.625. Left: BB fit; right: OTTB fit.

(A color version of this figure is available in the online journal.)

showing indeed that the OTTB is the preferred model, with an improvement in $C$-stat of $>30$. The BB and OTTB spectral fits are shown in Figure 7. Finally, the spectral analysis of the bursts with comparable flux values $\left(\sim 50\right.$ photons $\left.\mathrm{cm}^{-2} \mathrm{~s}^{-1}\right)$ and fluences $\left(\sim 13 \times 10^{-8} \mathrm{erg} \mathrm{cm}^{-2}\right)$ yielded significant differences of $\Delta C$-stat $>30$ for the best-fit models, leading us to the conclusion that this difference in spectral shape is intrinsic, and probably caused by a change in the burst emission process during the two periods.

We would like to point out that the Compt and $\mathrm{BB}+\mathrm{BB}$ models fit the burst spectra of this activity period equally well, with the exception of some events where fit parameters remained unconstrained. Using the successful $\mathrm{BB}+\mathrm{BB}$ fit results, we obtain a mean value for the cool BB temperatures of $5.1 \pm 0.1$ $\mathrm{keV}$ (width $0.6 \pm 0.1 \mathrm{keV}$ ), and for the hot BB temperatures of $14.7 \pm 0.2 \mathrm{keV}$ (width $0.9 \pm 0.1 \mathrm{keV}$ ). Only in the case of the second brightest burst 31 was the best-fit model the Compt model, with a $\Delta C$-stat of $\sim 40$; compared to an OTTB fit, the $\mathrm{BB}+\mathrm{BB}$ model in this case did fit equally well. This result is not conclusive, since the spectral slope of bright bursts could be affected by pulse pile-up effects as already pointed out in van der Horst et al. (2012). The spectral analysis of the brightest burst 24 was performed by excluding the saturated parts; nevertheless, none of the models, including the combined ones, provided a reasonable fit. Most probably, the whole emission period was affected by pulse pile-up and saturation effects.

To identify the model that best describes the SGR J1550-5418 burst data, we performed simulations similar to van der Horst et al. (2012) with RMFIT (4.0rc01). We selected the two bursts with similar brightness from the October and March-April periods, bursts 20 and 25, respectively. For each detector and each event, a set of 10,000 synthetic spectra was created. The background counts of these spectra are estimated from the real data whereas the source counts are computed from the function which was used to fit the real data folded with the detector response matrix (DRM). Poissonian noise was added to the sum of the source and background counts. During the fit process a synthetic background spectrum, with added Poisson fluctuations to 
each energy channel, was subtracted from the synthetic burst spectrum.

For burst 20 a set of 10,000 synthetic spectra was created with the OTTB function and its best parameters from the fit to the real data were used as the null hypothesis. The 10,000 spectra were then fitted with both the OTTB and a single BB function. The distribution of the difference in $C$-stat ( $\Delta C$-stat) was then compared to the $\Delta C$-stat obtained from the real burst data, which is $\Delta C$-stat OTтв-вв $_{1}=42$. There is not a single synthetic burst which exceeds this difference. In fact, the highest $\Delta C$-stat $_{\max }=6.3$. Subsequently, we conclude that statistical fluctuations cannot account for the difference in the statistic between OTTB and BB. Therefore, the null hypothesis is rejected $\left(P<10^{-4}\right)$ and we conclude that the BB function is the preferred model for this emission epoch.

The same line of reasoning was applied to the 2009 March event. However, contrary to the burst above, this time the BB was taken as the null hypothesis, i.e., 10,000 synthetic spectra were created using the $\mathrm{BB}$ model and its best-fit parameters from the real data as input model. For burst 25, we observe $\Delta C$-stat BB-оттв $=36$ in the real data. However, the maximum value of the simulated $C$-stat distribution is 10 . Similarly to what is observed above, we conclude that statistical fluctuations cannot account for the difference in $C$-stat and therefore we find the OTTB function to be the preferred model for this event.

\section{DISCUSSION}

Our main new finding in this work is that the observed GBM spectrum of the bursts in the energy range 8-200 keV has changed from being BB-like in the 2008 October active period to a broader, steeper OTTB-like form with less curvature during the 2009 March activity episode (see Table 2). In particular, the photon index below the peak, $\lambda$, that is defined through $d N / d E \propto E^{\lambda}$ has changed from $\lambda \sim 1$ during 2008 October to $\lambda \sim-1$ during 2009 March. In this context, it is interesting to note that during the most active bursting period of SGR J1550-5418, in 2009 January, the GBM burst spectra were typically not well fitted by a single BB spectrum, but were instead equally well fitted by an OTTB, Comptonized or $\mathrm{BB}+\mathrm{BB}$ spectrum (van der Horst et al. 2012). When including also Swift-XRT data in the spectral fit of those 2009 January bursts for which these were available, however, a $\mathrm{BB}+\mathrm{BB}$ spectrum is usually preferred (Lin et al. 2012) in which the cool $\mathrm{BB}$ component has a factor of $\sim 2$ or so smaller fluence than the hot BB component (interestingly enough, when fitted to a Comptonized spectrum, the implied values of $\lambda$ typically ranged between $\sim-1$ and $\sim 0$ ). The 2008 October bursts are those best fitted by a BB component of temperature $\sim 11-14 \mathrm{keV}$ and effective area $\sim 0.2-2 \mathrm{~km}^{2}$, which are similar to the hot BB component found for the 2009 January bursts. It is possible that a cool BB component could potentially still be present in the 2008 October bursts. If we assume a cool BB to hot BB flux ratio similar to that of the 2009 January bursts, then in order to avoid clear detection in the GBM spectra presented here the cool BB temperature typically needs to be below a few $\mathrm{keV}$, which would correspond to an effective area of $\gtrsim 10^{3} \mathrm{~km}^{2}$. In this scenario, both the hot and cold BB components in the 2008 October bursts would be near the cool end of the corresponding components from the $\mathrm{BB}+\mathrm{BB}$ spectral fits for the 2009 January bursts.

The differences between the spectroscopy of the bursts studied here and of the bursts in 2009 January clearly indicate evolutionary patterns on the timescale of a few months or so. This behavior might arise, for example, from a change in the details of the energy release or containment between different episodes. The rather small effective areas we obtain for the BB spectrum $\left(\sim 0.2-2 \mathrm{~km}^{2}\right)$ imply a small emission region, which is therefore probably close to the neutron star surface. Such a proximity to the surface would therefore tend to result in a relatively high effective opacity, due to large plasma densities. The opacity is mainly controlled by the scattering cross section of the ordinary or O-mode photons being near the Thomson value. O-mode photons are those where the photon electric field vector lies in the plane defined by their momenta $\mathbf{k}$ and the local magnetic field. Photons in the extraordinary polarization mode (or E-mode, where the photon electric field vector is normal to the local $\mathbf{k}-\mathbf{B}$ plane) experience a dramatically reduced scattering cross section that is suppressed because the photon energy is typically far below the cyclotron energy (e.g., Herold 1979). These E-mode photons contribute little to the overall opacity which, being high, results in a local quasi-thermodynamic equilibrium (i.e., LTE) that should drive the spectrum toward a BB or a $\mathrm{BB}+\mathrm{BB}$ form.

Radiative transfer effects in a strong magnetic field $(B \gg$ $B_{\mathrm{QED}}$ ) can cause a deviation from a pure BB spectrum (e.g., see Ulmer 1994; Lyubarsky et al. 2002) due to the increase in E-mode opacity with photon energy that enables lower energy (E-mode) photons to escape from a larger depth within the emission region, where the temperature (that is established by the O-mode photons) is higher, thus resulting in a softer than thermal spectral slope below the peak, where $d N / d E \propto E^{\lambda}$ with $\lambda \sim 0$. Somewhat harder low-energy spectral slopes might still be possible, e.g., due to resonant ion cyclotron absorption, which should be sensitive to the local value of the magnetic field. In order for such an absorption to reach sufficiently high photon energies, a rather high local value of the magnetic field is required in the emission region $\left(\gtrsim 10^{15} \mathrm{G}\right)$, which for SGR J1550-5418 is in excess of the surface dipole magnetic field strength (of $\sim 2.2 \times 10^{14} \mathrm{G}$ ) inferred from its $P \dot{P}$. Thus, this naturally suggests higher multipoles (e.g., Thompson et al. 2002). A strong local magnetic field from quadrupole and higher multipole configurations could effectively confine the hot emitting plasma to relatively small closed magnetic flux tubes near the stellar surface. This is consistent with the small effective areas inferred from our spectroscopic analysis. ${ }^{14}$ Thus, the spectral slope below the peak energy might be related to the local field strength and topology near the magnetic dissipation region that gives rise to the bursting episode. This might potentially be the factor that is common to different bursts within the same active period, but varies between different activity episodes, i.e., field topology evolves significantly on timescales of a month or so. It is also possible that the trigger from the crustal regions may relocate to different colatitudes during this evolution, thereby precipitating a sampling of disparate field topologies within the magnetospheric dissipation zones involved.

The broader OTTB-like spectra of the 2009 March bursts might reflect a Comptonized spectrum from an emitting region with a modest optical depth. As discussed, e.g., in Lin et al. (2011, 2012; Rybicki \& Lightman 1979), the simplest form of such a model yields $\lambda=1 / 2-\sqrt{9 / 4+4 / y_{B}}$ where $y_{B}=4 k T_{e} /\left(m_{e} c^{2}\right) \max \left[\tau_{B}, \tau_{B}^{2}\right]$ is the magnetic Compton

\footnotetext{
14 Some hint for the presence of higher multipoles might be found in the irregular shape of the pulse profiles of SGR J1550-5418, which change as a function of energy and time (e.g., Kaneko et al. 2010; Lin et al. 2012), but this is by no means conclusive.
} 
$y$-parameter, $\max \left[\tau_{B}, \tau_{B}^{2}\right]$ is the mean number of scatterings per photon by the hot electrons, and $\tau_{B}$ is the effective optical depth for scattering that in our case is significantly modified by the strong magnetic field (and is thus dubbed the magnetic optical depth). For SGR J1550-5418, the inferred peak energies for the 2009 March bursts, typically $E_{\text {peak }} \sim 30-45 \mathrm{keV}$, suggest $4 k T_{e} /\left(m_{e} c^{2}\right) \sim 0.23-0.35$, which would imply $\lambda \sim-0.8$ and $\sim-0.95$ for $\tau_{B} \sim 5$ and $\sim 10$, respectively. ${ }^{15}$ Thus, such modest values of $\tau_{B}$ would result in $y_{B} \gg 1$ so that $\lambda$ approaches the value of -1 , approximately coinciding with the lower energies of the inferred OTTB-like spectrum of the 2009 March bursts. Much larger optical depths would result in saturated Comptonization or true thermalization, and a spectrum closer to a $\mathrm{BB}$, though still generally different from a BB as mentioned above. Moreover, the 2009 March bursts are equally well fitted by a $\mathrm{BB}+\mathrm{BB}$ spectrum, so that in principal it is possible that the underlying spectra during all three bursting periods discussed above (2008 October, 2009 January, and 2009 March) are in fact $\mathrm{BB}+\mathrm{BB}$ or multi-BB, which was discussed in detail in van der Horst et al. (2012).

Different bursts within the same activity period may exhibit a relationship between the spectrum and the timing of the bursts. A given active period might be triggered by the yielding of the crust to magnetic stresses at a particular location on the neutron star. The magnetic field structure in that region could affect the details of the energy release and confinement of the hot plasma that is produced, and thereby influence the resulting spectrum of the bursts. If the emitting region is small and near the surface then it might be obscured during certain rotational phases (since the burst duration is smaller than the rotational period), hence resulting in a non-uniform distribution of bursts with the rotational phase, as was found by Lin et al. (2012). If the bursts indeed span a reasonable range of rotational phases, then it is likely that they sample substantially different viewing angles with respect to the well-localized emission region. This would then suggest that the viewing angle is not the dominant factor in determining the overall spectral shape.

This publication is part of the GBM/Magnetar Key Project (NASA grant NNH07ZDA001-GLAST, PI: C. Kouveliotou). Support for the German contribution to GBM was provided by the Bundesministerium für Bildung und Forschung (BMBF) via the Deutsches Zentrum für Luft und Raumfahrt (DLR) under contract number $50 \mathrm{QV}$ 0301. A.v.K. was supported by the Bundesministeriums für Wirtschaft und Technologie (BMWi) through DLR grant 50 OG 1101. C.K. and A.J.v.d.H. were partially supported by NASA grant NNH07ZDA001GLAST. M.G.B. acknowledges support from NASA through grant NNX10AC59A. E.G. and Y.K. acknowledge the support from the Scientific and Technological Research Council of Turkey (TÜBİTAK) through grant 109T755. L.L. is supported through the Post-Doctoral Research Fellowship of the Turkish Academy of Sciences (TÜBA). D.H. and A.L.W. acknowledge support from an NWO Vidi grant (PI: A. L. Watts).

\section{REFERENCES}

Abdo, A. A., Ackermann, M., Ajello, M., et al. 2010, ApJ, 725, L73 Bellm, E., Smith, D. M., \& Hurley, K 2009, GCN Circ., 8857 Bernardini, F., Israel, G. L., Stella, L., et al. 2011, A\&A, 529, A19

Bissaldi, E., von Kienlin, A., Lichti, G., et al. 2009, Exp. Astron., 24, 47 Camilo, F., Ransom, S. M., Halpern, J. P., \& Reynolds, J. 2007, ApJ, 666, L93

Connaughton, V., \& Briggs, M. S. 2009, GCN Circ., 8835

Duncan, R. C., \& Thompson, C. 1992, ApJ, 392, L9

Esposito, P., Israel, G. L., Zane, S., et al. 2008, MNRAS, 390, L34 Gavriil, F. P., Kaspi, V. M., \& Woods, P. M. 2002, Nature, 419, 142 Gavriil, F. P., Kaspi, V. M., \& Woods, P. M. 2004, ApJ, 607, 959 Gelfand, J. D., \& Gaensler, B. M. 2007, ApJ, 667, 1111

Göğüş, E., Kouveliotou, C., Woods, P. M., et al. 2001, ApJ, 558, 228 Göğüş, E., Woods, P. M., Kouveliotou, C., et al. 1999, ApJ, 526, L93 Göğüs, E., Woods, P. M., Kouveliotou, C., et al. 2000, ApJ, 532, L121 Golenetskii, S., Aptekar, R., Mazets, E., et al. 2009, GCN Circ., 8851 Gronwall, C., Holland, S. T., Markwardt, C. B., et al. 2009, GCN Circ., 8833 Herold, H. 1979, Phys. Rev. D, 19, 2868

Horne, J. H., \& Baliunas, S. L. 1986, ApJ, 302, 757

Israel, G. L., Esposito, P., Rea, N., et al. 2010, MNRAS, 408, 1387

Israel, G. L., Romano, P., Mangano, V., et al. 2008, ApJ, 685, 1114

Kaneko, Y., Göğüş, E., \& Kouveliotou, C. 2010, ApJ, 710, 1335

Kouveliotou, C., Dieters, S., Strohmayer, T., et al. 1998, Nature, 393, 235

Kouveliotou, C., Meegan, C. A., Fishman, G. J., et al. 1993, ApJ, 413, L101

Kouveliotou, C., Strohmayer, T., Hurley, K., et al. 1999, ApJ, 510, L115

Kouveliotou, C., von Kienlin, A., Fishman, G., et al. 2009, GCN Circ., 8915

Krimm, H. A., Beardmore, A. P., Burrows, D. N., et al. 2008a, GCN Circ., 8311

Krimm, H. A., Beardmore, A. P., Gehrels, N., et al. 2008b, GCN Circ., 8312

Lamb, R. C., \& Markert, T. H. 1981, ApJ, 244, 94

Lenz, P., \& Breger, M. 2005, Commun. Asteroseismol., 146, 53

Lin, L., Göğüş, E., Baring, M. G., et al. 2012, arXiv:1207.1434

Lin, L., Kouveliotou, C., Baring, M. G., et al. 2011, ApJ, 739, 87

Lyubarsky, Y., Eichler, D., \& Thompson, C. 2002, ApJ, 580, L69

Meegan, C., Lichti, G., Bhat, P. N., et al. 2009, ApJ, 702, 791

Mereghetti, S. 2008, A\&AR, 15, 225

Mereghetti, S., Götz, D., Weidenspointner, G., et al. 2009, ApJ, 696, L74

Ng, C.-Y., Kaspi, V. M., Dib, R., et al. 2011, ApJ, 729, 131

Paciesas, W. S., Meegan, C. A., von Kienlin, A., et al. 2012, ApJS, 199, 18

Paczynski, B. 1992, Acta Astron., 42, 145

Palmer, D. 2009, GCN Circ., 8901

Press, W. H., \& Rybicki, G. B. 1989, ApJ, 338, 277

Rea, N., Esposito, P., Krimm, H. A., et al. 2008, GCN Circ., 8313

Rybicki, G. B., \& Lightman, A. P. 1979, Radiative Processes in Astrophysics (New York, NY: Wiley), 393

Savchenko, V., Neronov, A., Beckmann, V., Produit, N., \& Walter, R. 2010, A\&A, 510, A77

Scargle, J.-D. 1982, ApJ, 263, 835

Schwarzenberg-Czerny, A. 1996, ApJ, 460, L107

Sugizaki, M., Mitsuda, K., Kaneda, H., et al. 2001, ApJS, 134, 77

Terada, Y., Tashiro, M., Urata, Y., et al. 2009, GCN Circ., 8845

Tiengo, A., Vianello, G., Esposito, P., et al. 2010, ApJ, 710, 227

Thompson, C., Lyutikov, M., \& Kulkarni, S. R. 2002, ApJ, 574, 332

Ulmer, A. 1994, ApJ, 437, L111

Usov, V. V. 1992, Nature, 357, 472

van der Horst, A. J., \& Briggs, M. S. 2008, GCN Circ., 8328

van der Horst, A. J., Kouveliotou, C., Gorgone, N. M., et al. 2012, ApJ, 749 , 122

Vaughan, S. 2005, A\&A, 431, 391

von Kienlin, A., \& Briggs, M. S. 2008, GCN Circ., 8315

von Kienlin, A., \& Connaughton, V. 2009, GCN Circ., 8838

Woods, P. M., \& Thompson, C. 2006, in Compact Stellar X-ray Sources, ed. W. H. G. Lewin \& M. van der Klis (Cambridge: Cambridge Univ. Press), 547

\footnotetext{
15 Note that relativistic corrections for such large temperatures, such as Klein-Nishina reductions, only influence these inferred indices to a modest extent; see Lin et al. (2011) and references therein.
} 